\title{
Evaluación de la calidad de vida en pacientes mexicanos con obesidad severa antes y después de cirugía bariátrica
}

\author{
Alejandra Albarrán-Sánchez, ${ }^{1}$ Claudia Ramírez-Rentería, ${ }^{2}$ Aldo Ferreira-Hermosillo, ${ }^{2 *}$ \\ Víctor Rodríguez-Pérez, ${ }^{3}$ Etual Espinosa-Cárdenas, ${ }^{1}$ Mario Molina-Ayala, ${ }^{1}$ Ilka Boscó-Gárate, ${ }^{4}$ y \\ Victoria Mendoza-Zubieta' \\ ${ }^{1}$ Instituto Mexicano del Seguro Social, Centro Médico Nacional Siglo XXI, Hospital de Especialidades, Servicio de Endocrinología; ${ }^{2}$ Instituto Mexicano \\ del Seguro Social, Centro Médico Nacional Siglo XXI, Hospital de Especialidades, Unidad de Investigación Médica en Enfermedades Endocrinas; \\ ${ }^{3}$ Clínica de Adherencia a Antirretrovirales y Polifarmacia, Clínica Especializada Condesa; ${ }^{4}$ Instituto Mexicano del Seguro Social, Centro Médico \\ Nacional Siglo XXI, Hospital de Especialidades, Unidad de Investigación Médica en Inmunoquímica. Ciudad de México, México
}

\section{Resumen}

Introducción: En México no se han utilizado los instrumentos Shorth Form 36 Items (SF-36) ni Baryatric Assesment Reporting Outcomes System (BAROS) para evaluar la calidad de vida (CV) antes y después de la cirugía bariátrica (CB). Objetivo: Describir los cambios en la CV con los cuestionarios SF-36 y BAROS, en pacientes con obesidad severa antes y después de la CB. Métodos: Se recolectaron los datos clínicos y antropométricos de pacientes sometidos a cirugía baríatrica entre 2015 y 2016. Se consideró con significación estadística una $p<0.05$. Resultados: Se analizaron 230 pacientes, 98 y 132 antes y después de la CB; la mayoría fue del sexo femenino (81\%). El índice de masa corporal inicial fue de $48 \mathrm{~kg} / \mathrm{m}^{2}(44-53)$. La CV medida con el SF-36 demostró un incremento en la puntuación del componente físico de 43 a $54.2(p<0.001)$ y en el componente mental, de 53.3 a 56.6 después de la CB. Con BAROS, en $98.5 \%$ se registraron resultados buenos a excelentes en la CV en los primeros tres meses. Conclusión: Al ser medida con los cuestionarios SF-36 y BAROS se definió que la $\mathrm{CV}$ de los pacientes mexicanos con obesidad severa mejora después de la CB.

PALABRAS CLAVE: Obesidad. Calidad de vida. Cuestionario. Cirugía bariátrica.

\section{Quality of life evaluation in Mexican patients with severe obesity before and after bariatric surgery}

\section{Abstract}

Introduction: In Mexico, neither the 36-item Short Form Health Survey (SF-36) nor the Bariatric Analysis and Reporting Outcome System (BAROS) instruments have been used to assess quality of life (QoL) before and after bariatric surgery (BS). Objective: To describe changes in QoL using the SF-36 and BAROS questionnaires in patients with severe obesity before and after BS. Methods: Clinical and anthropometric data of patients undergoing bariatric surgery between 2015 and 2016 were collected. Statistical significance was considered with a p-value $<0.05$. Results: 230 patients were analyzed, 98 before and 132 and after BS; most were females (81\%). Initial body mass index was 48 kg/m² (44-53). SF-36-measured QoL showed an increase in the physical component score from 43 to 54.2 points $(p<0.001)$, and in the mental component, from 53.3 to 56.6 points after BS. With BAROS, $98.5 \%$ showed good to excellent QoL results within the first three months after BS.

Correspondencia:

*Aldo Ferreira-Hermosillo

E-mail: aldo.nagisa@gmail.com CC BY-NC-ND (http://creativecommons.org/licenses/by-nc-nd/4.0/)
Fecha de recepción: 13-06-2020

Fecha de aceptación: 09-07-2020

DOI: 10.24875/GMM.20000385
Gac Med Mex. 2021;157:67-73

Disponible en PubMed

www.gacetamedicademexico.com 
Conclusion: When measured with the SF-36 and BAROS questionnaires, QoL of Mexican patients with severe obesity was found to improve after BS.

KEY WORDS: Obesity. Quality of life. Questionnaire. Bariatric surgery.

\section{Introducción}

La Organización Mundial de la Salud reporta que $3 \%$ de la población mexicana tiene obesidad severa o mórbida, definida como un índice de masa corporal $\left(\mathrm{IMC}, \mathrm{kg} / \mathrm{m}^{2}\right)>40 \mathrm{~kg} / \mathrm{m}^{2}{ }^{1,2}$ La cirugía bariátrica (CB) es el único tratamiento para la obesidad severa que ha logrado una reducción de peso a mediano plazo significativa, constante y que consigue controlar enfermedades asociadas a la obesidad. ${ }^{3} \mathrm{El}$ incremento en el IMC se relaciona con menor calidad de vida (CV). No existe información suficiente del efecto de la cirugía bariátrica en la CV de la población mexicana. ${ }^{4-6}$

Medir la CV no es sencillo y para hacerlo existen varios instrumentos. El cuestionario BAROS (Baryatric Assesment Reporting Outcomes System) es un instrumento sencillo, validado y específico para pacientes a quienes se realizó cirugía bariátrica, que evalúa el éxito de la cirugía según la pérdida del exceso de peso, así como mejoría de las comorbilidades y de la CV. ${ }^{6-8}$ Favretti et al..$^{9}$ reportaron resultados de buenos a excelentes en $48 \%$ de la población, mientras que Velásquez Zambrano ${ }^{10}$ registró resultados excelentes en los primeros seis meses posteriores a la cirugía bariátrica en $31.7 \%$ de los pacientes y en $42.9 \%$ a los tres años. ${ }^{8,9}$

El SF-36 (Short Form-36 Health Survey) es el instrumento de medición de CV general más usado en el mundo y también está validado en población mexicana. Es autoaplicable y se califica en una escala de 0 a 100, en la cual una mayor puntuación significa mejor calidad de vida.11,12 Se ha demostrado que posterior a la cirugía bariátrica existe mejoría, principalmente en el componente físico; sin embargo, existe poca información en México., ${ }^{5,13-15}$

El propósito del estudio fue conocer con el cuestionario SF-36, la CV de una cohorte de pacientes mexicanos antes y después de la cirugía bariátrica y con el cuestionario BAROS después de la intervención quirúrgica.

\section{Métodos}

Entre febrero de 2015 y febrero de 2016 se invitó a participar a los candidatos a CB $(n=98)$ que acudieron a la Clínica de Obesidad y a quienes estaban en seguimiento posterior a la cirugía en diferentes tiempos de tres meses a cinco años $(n=132)$. Previa lectura y firma del consentimiento informado (registro ante el Comité Local de Ética en Investigación en Salud 3601 del IMSS: R-2016-3601-27), a todos los pacientes se les aplicó el cuestionario SF-36 antes y después de la cirugía y el cuestionario BAROS después de la intervencion quirúrgica. Se evaluó y registró su edad, antecedentes de enfermedades, datos antropométricos y los resultados de sus cuestionarios.

\section{Análisis estadístico}

Para el análisis estadístico se utilizó el programa SPSS versión 23. Las variables cuantitativas se describieron como medianas con rangos intercuartíliticos debido a su distribución. Las variables cualitativas se describieron como frecuencias o porcentajes. Para el análisis bivariado se utilizó $U$ de Mann-Whitney (para la evaluación entre dos grupos) o prueba de ANOVA (para tres grupos). Las variables cualitativas se evaluaron mediante prueba de chi cuadrada. Se consideró con significación estadística una $p<0.05$.

\section{Resultados}

Se incluyó a 230 pacientes con mediana de edad de 45 años (rango intercuartílico [RIC] de 37-50 años), $81 \%$ mujeres; el peso inicial fue de $142 \mathrm{~kg}$ en los hombres y de $125 \mathrm{~kg}$ en las mujeres. El IMC inicial fue de 48 (44-53); $53 \%$ tenía un IMC de 40-49.9; $30 \%$, de 50-59.9 (superobesidad); y $9 \%$, IMC mayor a 60 (súper superobesidad). En la población total, el plan nutricional logró una reducción de peso de 126 a $110 \mathrm{~kg}(\mathrm{p}<0.001)$ y el último peso registrado posterior a la cirugía bariátrica fue $79 \mathrm{~kg}(67-93 \mathrm{~kg})$. El porcentaje de exceso de peso perdido (\% EPP) durante el primer año posterior a cirugía fue $59 \%$. Como se observa en la Tabla 1, las principales comorbilidades previas a la cirugía fueron hipertensión arterial sistémica, prediabetes, diabetes mellitus, dislipidemia y apnea obstructiva del sueño. A la mayoría de los pacientes se les realizó derivación gástrica laparoscópica. 
Tabla 1. Características generales de población con obesidad severa

\begin{tabular}{|c|c|c|}
\hline \multirow[t]{2}{*}{ Parámetro } & \multicolumn{2}{|c|}{ Valor $(n=230)$} \\
\hline & Mediana & RIC \\
\hline Edad (años) & 45 & $37-50$ \\
\hline Peso inicial (kg) & 126 & $113-140$ \\
\hline IMC $\left(\mathrm{kg} / \mathrm{m}^{2}\right)$ & 48 & $44-53$ \\
\hline Peso prequirúrgico (kg) & 110 & $106-125$ \\
\hline \% EPP a los 12 meses & 59 & $54-72$ \\
\hline \multirow[t]{2}{*}{ \% EPP a los 4 años } & 84 & $77-88$ \\
\hline & $\mathrm{n}$ & $\%$ \\
\hline Mujeres & 146 & 81 \\
\hline Diabetes mellitus tipo 2 & 56 & 25 \\
\hline Prediabetes & 93 & 42 \\
\hline Hipertensión arterial sistémica & 112 & 49 \\
\hline Apnea obstructiva del sueño & 59 & 26 \\
\hline Dislipidemia & 76 & 34 \\
\hline Osteoartritis & 40 & 18 \\
\hline Trombosis venosa profunda & 12 & 5 \\
\hline Insuficiencia venosa periférica & 37 & 17 \\
\hline Hipotiroidismo & 52 & 24 \\
\hline Trastorno psiquiátrico & 50 & 26 \\
\hline Esteatosis hepática no alcohólica & 25 & 12 \\
\hline Hiperuricemia & 24 & 12 \\
\hline Enfermedad de reflujo gastroesofágico & 8 & 4 \\
\hline Gastrectomía vertical & 3 & 2 \\
\hline Derivación gástrica en $Y$ de Roux & 57 & 39 \\
\hline Derivación gástrica de una anastomosis & 86 & 59 \\
\hline
\end{tabular}

\section{Instrumento general SF-36}

Las medianas de las sumatorias de los componentes físico y mental antes de la cirugía fueron 43 puntos $(\mathrm{RIC}=36-50)$ y 53.3 puntos $(\mathrm{RIC}=42.1-57.7)$, respectivamente. No hubo diferencias entre los estratos de IMC ni en los sexos en la puntuación del SF-36 prequirúrgico. El aumento de la puntuación del SF-36 se observó de los tres a 12 meses posteriores a la $\mathrm{CB}$; no se registró aumento significativo de la puntuación después de los 12 meses de la cirugía. Se observaron diferencias en los componentes físico y
Tabla 2. Resultados del cuestionario SF-36 antes y después de la cirugía bariátrica

\begin{tabular}{|l|c|c|c|}
\hline Variable & $\begin{array}{c}\text { Puntuación } \\
\text { preoperatoria } \\
(\mathbf{n = 9 8 )}\end{array}$ & $\begin{array}{c}\text { Puntuación } \\
\text { posoperatoria } \\
(\mathbf{n = 1 3 2})\end{array}$ & $p^{*}$ \\
\hline Munción física & $52.9(36.2-75)$ & $57(52-57.1)$ & 0.778 \\
\hline Rol físico & $56.2(32-100)$ & $56.2(56.2-56.2)$ & 0.228 \\
\hline Dolor corporal & $51.6(37-62.7)$ & $51.6(46.5-62.7)$ & 0.605 \\
\hline Salud general & $46.6(39.5-62)$ & $57.9(50.2-62.6)$ & $<0.001$ \\
\hline Vitalidad & $53.8(44.3-65.6)$ & $60.9(53.8-65.6)$ & 0.001 \\
\hline Función social & $57.1(46.3-87.5)$ & $57.1(46.3-57.1)$ & 0.001 \\
\hline Rol emocional & $55.3(55.3-100)$ & $55.3(55.3-55.3)$ & $<0.001$ \\
\hline Salud mental & $59.7(50-76)$ & $56.1(48.2-61.8)$ & 0.014 \\
\hline Componente físico & $43(36-50.0)$ & $54.2(48.2-61.8)$ & $<0.001$ \\
\hline Componente mental & $53.3(42.1-57.7)$ & $56.6(50.8-60.8)$ & 0.002 \\
\hline *p<0.005 evaluada mediante U de Mann-Whitney. & &
\end{tabular}

componente mental al comparar antes y después de la cirugía (Tabla 2). Se observaron cambios significativos en el área salud general y vitalidad después de la CB.

Cada uno de los dominios de los componentes físico, psicológico, social y la puntuación total del SF-36 de los pacientes a quienes se realizó $\mathrm{CB}$, clasificados por estratos del IMC inicial se compararon con los resultados de la CV en población general sana publicados por Zúñiga et al.:12 se observó que, en todos los componentes, la puntuación era baja aún después de la CB (Tablas 3 y 4).

\section{Instrumento específico BAROS}

En $98.5 \%$ de los pacientes, después de la cirugía se obtuvo resultado bueno, muy bueno y excelente, lo cual se mantiene hasta cinco años posterior a la cirugía. En CV del cuestionario BAROS, los resultados fueron regular en 14 pacientes (10\%), buena en $23(18 \%)$ y muy buena en 94 (72 \%) (Figura 1).

Respecto a la valoración de las comorbilidades con el cuestionario BAROS, se identificó mejoría en ocho pacientes (6\%), que no existieron cambios en 13 $(10 \%)$, una comorbilidad mayor resuelta y otras mejoradas en 18 pacientes (14\%) y todas las comorbilidades mayores resueltas y otras mejoradas en 92 pacientes $(70 \%)$. El exceso de peso perdido fue de bueno a excelente y se correlacionó con la 
Tabla 3. Comparación de los resultados del cuestionario SF-36 antes de la cirugía bariátrica en diferentes grados de obesidad con los de población general sana

\begin{tabular}{|c|c|c|c|c|c|}
\hline Componente de salud & Dominio & Población general $(n=12)$ & IMC $40-50(n=123)$ & IMC $50-60(n=70)$ & $I M C>60(n=22)$ \\
\hline \multirow[t]{5}{*}{ Físico } & Función física & $94.6 \pm 8.6$ & $54 \pm 18^{+}$ & $49 \pm 11^{\dagger}$ & $53 \pm 15.6^{+}$ \\
\hline & Rol físico & $94.5 \pm 15.4$ & $54.2 \pm 23^{\dagger}$ & $57.4 \pm 17^{\dagger}$ & $52 \pm 26^{\dagger}$ \\
\hline & Dolor corporal & $88.6 \pm 15.4$ & $52 \pm 17^{\dagger}$ & $53.5 \pm 14^{\dagger}$ & $50.2 \pm 13^{+}$ \\
\hline & Salud general & $72.9 \pm 15.5$ & $52.9 \pm 13^{\dagger}$ & $54.5 \pm 11^{\dagger}$ & $53.7 \pm 12^{\dagger}$ \\
\hline & Vitalidad & $81 \pm 11.6$ & $54.7 \pm 13^{\dagger}$ & $58.9 \pm 11^{\dagger}$ & $55.3 \pm 16^{\ddagger}$ \\
\hline \multirow[t]{2}{*}{ Psicológico } & Rol emocional & $83.4 \pm 25.1$ & $54.5 \pm 22^{\dagger}$ & $56.4 \pm 20^{\dagger}$ & $64 \pm 25^{\dagger}$ \\
\hline & Salud mental & $79.6 \pm 13.9$ & $55.8 \pm 13^{\dagger}$ & $56.9 \pm 15^{\dagger}$ & $58 \pm 15^{\dagger}$ \\
\hline Social & Función social & $86.9 \pm 15.6$ & $54.5 \pm 16^{\dagger}$ & $56.2 \pm 17^{\dagger}$ & $59.3 \pm 15^{\dagger}$ \\
\hline
\end{tabular}

Los valores se presentan como media \pm desviación estándar.

Los obeliscos indican la significación de comparar cada resultado con la población general: ${ }^{t} p<0.001$, ${ }^{\ddagger} p<0.05$ utilizando prueba T de Student.

Tabla 4. Comparación de los resultados del cuestionario SF-36 después de la cirugía bariátrica con los de población general sana

\begin{tabular}{|c|c|c|c|c|}
\hline $\begin{array}{l}\text { Componente } \\
\text { de salud }\end{array}$ & Dominio & $\begin{array}{l}\text { Población } \\
\text { general } \\
(n=12)\end{array}$ & Posoperados & $\mathbf{p}^{*}$ \\
\hline \multirow[t]{5}{*}{ Físico } & $\begin{array}{l}\text { Función } \\
\text { física }\end{array}$ & $94.6 \pm 8.6$ & $53.9 \pm 8.4$ & $<0.001$ \\
\hline & Rol físico & $94.5 \pm 15.4$ & $53.5 \pm 8.8$ & $<0.001$ \\
\hline & $\begin{array}{l}\text { Dolor } \\
\text { corporal }\end{array}$ & $88.6 \pm 15.4$ & $52.6 \pm 10.3$ & $<0.001$ \\
\hline & $\begin{array}{l}\text { Salud } \\
\text { general }\end{array}$ & $72.9 \pm 15.5$ & $55.7 \pm 8.4$ & $<0.001$ \\
\hline & Vitalidad & $81 \pm 11.6$ & $58.5 \pm 9.4$ & $<0.001$ \\
\hline \multirow[t]{2}{*}{ Psicológico } & $\begin{array}{l}\text { Rol } \\
\text { emocional }\end{array}$ & $83.4 \pm 25.1$ & $51.9 \pm 10$ & $<0.001$ \\
\hline & $\begin{array}{l}\text { Salud } \\
\text { mental }\end{array}$ & $79.6 \pm 13.9$ & $54.2 \pm 10.2$ & $<0.001$ \\
\hline Social & $\begin{array}{l}\text { Función } \\
\text { social }\end{array}$ & $86.9 \pm 15.6$ & $52.4 \pm 9.9$ & $<0.001$ \\
\hline
\end{tabular}

calificación del cuestionario BAROS $(r=0.421$ $\mathrm{p}<0.001)$. En el apartado de calidad de vida hubo mejoría en todos los componentes, excepto en el componente sexual.

\section{Discusión}

Este es uno de los primeros estudios que evalúa la $\mathrm{CV}$ en pacientes mexicanos en un programa de $\mathrm{CB}$ o que ya fueron operados, mediante el cuestionario SF-36 y BAROS. En nuestro estudio observamos que existió mejoría en la CV, predominantemente en el componente físico, lo cual concuerda con los resultados de otras investigaciones. ${ }^{13,14}$ Este cambio podría estar relacionado con la mejoría de las comorbilidades asociadas a la obesidad y a la disminución significativa de peso. Observamos descenso en la puntuación del dominio de salud mental, que podría estar influido por factores no evaluados, como la personalidad, trastornos de conducta alimentaria, depresión, ansiedad, trastornos de personalidad, aspectos relacionados con el entorno social, parámetros de autoestima y expectativas diferentes de la cirugía bariátrica, entre otros. ${ }^{16}$

Se ha observado que los componentes físico y mental de la CV se reducen conforme se incrementa el IMC, debido a que la obesidad causa o agrava diferentes condiciones de salud que van desde las enfermedades cardiovasculares hasta patologías musculoesqueléticas, además de que disminuye la esperanza de vida en general. ${ }^{4}$ Sin embargo, al comparar la CV de los pacientes después de la cirugía con la de población sana observamos puntuaciones bajas a pesar de la reducción del IMC, lo cual traduce que los pacientes con obesidad siguen percibiendo que su salud física y mental no es adecuada. Es posible que esta percepción se deba a que después de la intervención quirúrgica continúan en seguimiento periódico con el médico (lo cual puede ser percibido como continuar enfermo o ser un enfermo "crónico"), el estrés psicológico causado por los cambios al estilo de vida, la percepción del entorno, la autoestima, la 


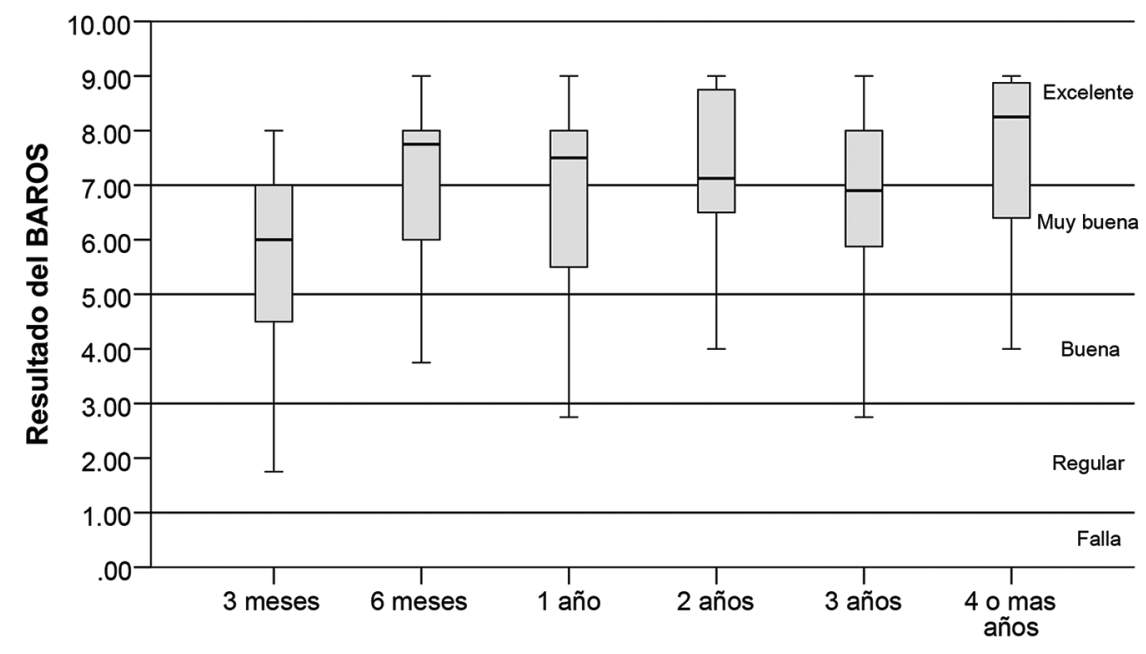

Tiempo en el que se determinó el resultado de BAROS

Figura 1. Resultado del Cuestionario BAROS (Bariatric Assessment Reporting Outcomes System). Se muestran los resultados después de la cirugía en diferentes tiempos, desde 3 meses a 4 años.

aceptación de la imagen corporal la presencia de flacidez en la piel, entre otros factores. ${ }^{16,17}$ Esta falta de mejoría de los niveles reportados en la población general se ha observado en enfermedades con comportamiento crónico como diabetes, enfermedad renal terminal, artritis reumatoide y cáncer. $^{18}$ Este es un resultado importante para sustentar el seguimiento a largo plazo posterior a la cirugía por parte de un equipo multidisciplinario.

El cuestionario BAROS indicó resultados excelentes en la mayoría de los casos. Los autores consideramos que esto se asocia a la mejoría física relacionada con el exceso de peso perdido. Por otra parte, los aspectos sociales, mentales y sexuales abordados en el cuestionario no mostraron esta mejoría, lo cual es consistente con otros estudios..$^{10,15} \mathrm{Tal}$ como se observa con nuestros resultados, el tratamiento de esta enfermedad crónica debe ser integral antes y después de la $\mathrm{CB}$, no solo de la condición de salud física y metabólica, sino también de los aspectos psicológicos, que pueden influir en la reincorporación a la sociedad, vida laboral y familiar. En cuanto al aspecto laboral, la mayor parte de los pacientes en nuestro estudio fueron jóvenes económicamente activos. Kantarovich $\mathrm{K}$ et al. ${ }^{19}$ observaron que el aumento de la CV dos años después de la CB predice mejoría en la productividad y reduce la discapacidad laboral, de tal manera que todas las intervenciones destinadas a apoyar y mantener la CV, especialmente las relacionadas con la salud mental en el periodo posoperatorio incrementarán la mejoría del funcionamiento relacionado con el empleo.

En nuestro estudio, $26 \%$ de la población tenía un trastorno psiquiátrico preexistente, lo cual puede influir en los resultados. Müller et al. observaron que después de cuatro años de la cirugía, los trastornos mentales estaban relacionados con ganancia de peso posterior a esta, ${ }^{20}$ similar a lo que hemos encontrado previamente en nuestra población. ${ }^{21}$ Consideramos que, aun cuando en este estudio el porcentaje de exceso de peso perdido en el primer año fue adecuado (disminución de más de $50 \%$ ), faltan estudios prospectivos que evalúen el impacto de depresión, ansiedad y calidad de vida a largo plazo, así como sus efectos en la reganancia de peso, ya que estos factores pueden influir en las modificaciones de conductas, como la necesidad de realizar actividad física, y en la adherencia al plan nutricional en el periodo posoperatorio. ${ }^{22}$

En el posoperatorio existen cambios neuroendocrinos (eje hipotálamo-hipófisis), involucrados en la aparición de alteraciones emocionales, por ejemplo, la reducción en los niveles de neuropéptidos provenientes del tracto gastrointestinal que regulan la acción central del neuropéptido $\mathrm{Y}$, que se asocian a la aparición de depresión y adicciones y al incremento en el impulso hedónico por los alimentos. ${ }^{23}$ Aún faltan estudios que combinen enfoques sociológicos y psicológicos con medidas objetivas en la elección de alimentos después de la CB. ${ }^{24}$ 
Este estudio nos permitió evaluar los resultados de la $\mathrm{CB}$ en cuanto a reducción de peso, mejoría de comorbilidades y $\mathrm{CV}$, que fueron similares a los reportados en otros centros internacionales. En México, la obesidad es un problema de salud pública y el acceso a la CB es limitado, debido a que son pocos los sitios que cuentan con equipo multidisciplinario. Es necesaria la creación de nuevas clínicas de obesidad a lo largo del país, considerando los beneficios en cuanto a la remisión de comorbilidades, mejoría de la calidad de vida en el componente físico y mental, así como la reducción en la mortalidad de pacientes en edad productiva. Estas clínicas deben tener un seguimiento posterior a la $\mathrm{CB}$, considerando, además de la mejoría en los parámetros clínicos o bioquímicos, la mejoría de la CV, ya que es escasa la información de los factores que determinan la $\mathrm{CV}$ en los pacientes mexicanos.

Una de las principales limitaciones del estudio consiste que al ser un estudio transversal no se está comparando a las poblaciones de manera longitudinal contra sus propios resultados basales. Sin embargo, hasta el momento ningún otro estudio prospectivo ha evaluado CV en pacientes mexicanos. A pesar de esto, las características basales de los pacientes que fueron operados son similares a las de los pacientes antes de la cirugía, lo cual sugiere la posibilidad de que los resultados sean aplicables a todos los pacientes en condiciones similares. El uso del cuestionario BAROS, diseñado únicamente para evaluar los resultados posteriores a la cirugía y que no requiere una línea basal, ayuda también a que se evalúe si los resultados de la $\mathrm{CV}$ en el posoperatorio son concordantes.

Debemos considerar que los objetivos de los cuestionarios no son equivalentes. El cuestionario SF-36 trata la calidad de vida desde el punto de vista físico y mental del paciente, lo cual puede variar en una población o según la enfermedad, mientras que BAROS da un gran peso a los resultados técnicos de la cirugía bariátrica. Ambos están sujetos a la adecuada estandarización, traducción y aplicación para su interpretación. Podemos apreciar como BAROS integró resultados buenos o excelentes para la mayor parte de los pacientes a corto plazo, los cuales se mantienen en rangos altos aun en pacientes evaluados años después de la cirugía, mientras que SF-36 indicó que los pacientes tuvieron mala calidad de vida en todos los aspectos antes de la cirugía, pero que después del procedimiento percibieron una mejoría en el aspecto físico, pero no en el mental.
Aunque es necesario realizar un estudio de cohorte con estos pacientes, a quienes se aplique cuestionarios basales y posquirúrgicos, la cantidad de sujetos que se requiere para lograr una muestra significativa para el tiempo de seguimiento evaluado en este estudio probablemente implicaría una inversión de recursos importante. Esta es una de las razones por las cuales no existen numerosas publicaciones de este tipo en la literatura mundial.

\section{Conclusiones}

La puntuación con los instrumentos de calidad de vida SF-36 y BAROS en los pacientes con obesidad severa mejoró después de la cirugía bariátrica. Sin embargo, a pesar de los beneficios rápidos y persistentes de la cirugía, los pacientes a quienes se les realizó cirugía bariátrica tuvieron pobre $\mathrm{CV}$, similar a lo que sucede en otras enfermedades crónicas.

\section{Conflictos de intereses}

Los autores declaran no tener conflicto de intereses alguno.

\section{Financiamiento}

Los autores no recibieron patrocinio para llevar a cabo este artículo.

\section{Responsabilidades éticas}

Protección de personas y animales. Los autores declaran que los procedimientos seguidos se conformaron a las normas éticas del comité de experimentación humana responsable y de acuerdo con la Asociación Médica Mundial y la Declaración de Helsinki.

Confidencialidad de los datos. Los autores declaran que siguieron los protocolos de su centro de trabajo sobre la publicación de datos de pacientes.

Derecho a la privacidad y consentimiento informado. Los autores obtuvieron el consentimiento informado de los pacientes o sujetos referidos en el artículo. Este documento obra en poder del autor de correspondencia.

\section{Bibliografía}

1. World Health Organization [Internet]. Suiza: Obesity and overweight 2015; 2020.

2. Barquera S, Campos-Nonato I, Hernández-Barrera L, Pedroza A, Rivera-Dommarco JA. Prevalence of obesity in Mexican adults 20002012. Salud Publica Mex. 2013;55:S151-S160. 
3. Barrera-Cruz A, Rodríguez-González A, Molina-Ayala MA. The current state of obesity in Mexico. Rev Med Inst Mex Seguro Soc. 2013:51:292-299.

4. UI-Haq Z, Mackay DF, Fenwick E, Pell JP. Meta-analysis of the association between body mass index and health-related quality of life among adults, assessed by the SF-36. Obesity (Silver Spring); 2013;21: E322-E327.

5. Rivas A, Ocejo S, Sierra M. Evaluación de la calidad de vida en pacientes sometidos a cirugía de obesidad mórbida. Medicina Universitaria. 2009;11:243-246

6. Chang CY, Hung CK, Chang YY, Tai CM, Lin JT, Wang JD. Health-related quality of life in adult patients with morbid obesity coming for bariatric surgery. Obes Surg. 2010;20:1121-1127.

7. Consenso Argentino de Nutrición en Cirugía Bariátrica. Argentina: Congreso Internacional de Cirugía Bariátrica y Metabólica; 2011

8. Puzziferri N, Austrheim-Smith IT, Wolfe BM, Wilson SE, Nguyen NT. Three-year follow-up of a prospective randomized trial comparing laparoscopic versus open gastric bypass. Ann Surg. 2006;243:181-188.

9. Favretti F, Cadiere GB, Segato G, Busetto L, Loffredo A, Vertruyen M, et al. Bariatric analysis and reporting outcome system (BAROS) applied to laparoscopic gastric banding patients. Obesity Surgery. 1998;8:500-504.

10. Velásquez-Zambarana JG, Miranda-Fontalvo A, Pulgar-Emanuelli MA, Araujo-Zarate PS, Salazar-Maestre CJ. Evaluación de la calidad de vida en pacientes obesos y cirugía bariátrica. Rev Cienc Biomed. 2014;5:79-87.

11. Velarde-Jurado E, Ávila-Figueroa C. Evaluación de la calidad de vida. Salud Publica Mex. 2002;44:349-361.

12. Zúñiga MA, Carrillo-Jiménez GT, Fos PJ, Gandek B, Medina-Moreno MR Evaluación del estado de salud con la Encuesta SF-36: resultados preliminares en México. Salud Publica Mex. 1999:41:110-118.

13. van der Hofstadt CJ, Escribano-Cubas S, Tirado-González S, Pérez-Martínez E, Ortiz Sebastián S, Estrada Caballero JL, et al. Evolución de la calidad de vida a los 24 meses de seguimiento en pacientes sometidos a cirugía bariátrica: comparación entre el bypass gástrico y la gastrectomía vertical tubular. En: Anales del Sistema Sanitario de Navarra. España: Gobierno de Navarra/Departamento de Salud; 2017.
14. Raaijmakers LCH, Pouwels S, Thomassen SEM, Nienhuijs SW. Quality of life and bariatric surgery: a systematic review of short-and long-term results and comparison with community norms. Eur J Clin Nutr. 2017;71:441-449.

15. De Queiroz C, Sallet JA, de Barros e Silva PGM, de Souse-Queiroz LG, Pimentel JA, Sallet PC. Application of BAROS'questionnaire in obese patients undergoing bariatric surgery with 2 years of evolution. Arq Gastroenterol. 2017;54:60-64.

16. Peterhänsel $C$, Nagl M, Wagner B, Dietrich A, Kersting A. Predictors of changes in health-related quality of life 6 and 12 months after a bariatric procedure. Obes Surg. 2017;27:2120-2128.

17. Paul MA, Opyrchał J, Knakiewicz M, Jaremków P, Duda-Barcik $Ł$, Ibrahim AM, et al. The long-term effect of body contouring procedures on the quality of life in morbidly obese patients after bariatric surgery. PloS One. 2020;15:e0229138.

18. Murillo YA, Almagro RM, Campos-González ID, Cardiel MH. Health related quality of life in rheumatoid arthritis, osteoarthritis, diabetes mellitus, end stage renal disease and geriatric subjects. Experience from a General Hospital in Mexico. Reumatol Clin. 2015;11:68-72.

19. Kantarovich, K, Wnuk S, Cassin S, Hawa R, Sockalingam S. Employment outcomes 2 years after bariatric surgery: relationship to quality of life and psychosocial predictors. Obes Surg. 219;29:2854-2861.

20. Müller M, Nett PC, Borbély YM, Buri C, Stirnimann G, Laederach K, et al. Mental illness has a negative impact on weight loss in bariatric patients: a 4-year follow-up. J Gastrointest Surg. 2019;23:232-238.

21. Cadena-Obando D, Ramírez-Rentería C, Ferreira-Hermosillo A, Albarrán-Sánchez A, Sosa-Eroza E, Molina-Ayala M, et al. Are there really any predictive factors for a successful weight loss after bariatric surgery? BMC Endocr Disord. 2020;20:20.

22. Gerlach G, Herpertz S, Loeber S. Personality traits and obesity: a systematic review. Obes Rev. 2015;16:32-63.

23. Morales-Medina JC, Dumont Y, Quirion R. A possible role of neuropeptide $Y$ in depression and stress. Brain Res. 2010;1314:194-205.

24. Hansen TT, Jakobsen TA, Nielsen MS, Sjödin A, Le Roux CW, Schmidt JB. Hedonic changes in food choices following Roux-en-Y gastric bypass. Obes Surg. 2016;26:1946-1955 